\title{
La simulation : développement d'un outil pédagogique devenu un paradigme en médecine d'urgence
}

\author{
Simulation: development of a teaching tool that has become a paradigm in emergency medicine
}

\author{
D.A. Ghazali · E. Casalino \\ (C) SFMU et Lavoisier SAS 2018
}

La pédagogie médicale constitue l'apprentissage du savoir, du savoir-faire et du savoir-être. Cet enseignement repose dans son versant pratique de plus en plus sur la simulation. Cet outil pédagogique devient prépondérant pour les disciplines médicales dites à « haut risque » comme la médecine d'urgence. La simulation en médecine d'urgence a connu un essor outre-Atlantique pour devenir un paradigme dans les années 2000 [1]. Depuis le rapport de la Haute Autorité de santé (HAS) [2], la simulation connaît au cours de cette décennie la même évolution en France. Elle permet d'améliorer les compétences techniques et non techniques individuelles et en équipes pluriprofessionnelles [3]. La gestion de situation de crise (Crisis Resource Management, CRM) pour la sureté d'un patient dépend en effet de la réalisation de gestes techniques et thérapeutiques, compris dans un algorithme de prise en charge (c'est-à-dire séquence dans laquelle ils sont priorisés), et d'une interaction entre les différents membres de l'équipe impliqués [4].

Dans ce numéro des Annales françaises de médecine d'urgence, Allain, et al. [5] rapportent une enquête observationnelle nationale intéressante sur la place de la simulation dans la formation initiale des urgentistes. Cet article présente en effet l'intérêt de montrer l'évolution favorable de la simulation comme outil pédagogique prépondérant de l'enseignement universitaire initial, et en particulier dans la médecine d'urgence. Il permet également de constater que certaines facultés de médecine ne sont pas encore toutes dotées de cen-

\footnotetext{
D.A. Ghazali $(\bowtie)$

Centre de simulation, université Paris-Diderot, site Pajol,

20, rue du Département, F-75018 Paris, France

e-mail : aiham@hotmail.com

E. Casalino

Université Paris-Diderot, Sorbonne-Paris-Cité, EA 7334, site Villemin, 10, avenue de Verdun, F-75010 Paris, France

D.A. Ghazali · E. Casalino

Département des urgences et Smur de Bichat et Beaujon, groupe hospitalier Paris Nord-Val-de-Seine,

46, rue Henri-Huchard, F-75018 Paris, France
}

tres de simulation ou du moins d'un accès à l'un d'entre eux. La deuxième idée qui peut être soulevée par cette étude, c'est l'inhomogénéité des moyens techniques dont disposent les centres de simulation pour l'enseignement (modèles procéduraux, mannequins basse et haute fidélité, jeux sérieux et outils de réalité virtuelle, patient standardisé...). Plus spécifique à notre discipline, cette analyse permet de constater que dans certaines facultés ayant un centre de simulation, les internes de médecine d'urgence n'y ont pas accès et que les champs d'enseignement sont disparates (préhospitalier, régulation, urgences hospitalières adultes ou pédiatriques...). En revanche, outre ces axes d'amélioration mis en exergue par cette étude, et à l'heure où la médecine d'urgence est devenue une spécialité [6], il faut aller plus loin dans la réflexion pour le développement de l'enseignement par simulation, voulu par les coordonnateurs du Diplôme d'études spécialisées (DES) de médecine d'urgence.

Tout d'abord, il est nécessaire d'avoir des échelles validées d'évaluation des performances réalisées. Celles-ci sont encore peu développées, en particulier dans le domaine des compétences techniques [7-10]. Cette remarque renforce un premier axe d'évolution à préconiser pour les centres de simulation : la recherche en pédagogie par simulation et notamment pour la médecine d'urgence. La recherche est diversifiée et peut concerner de manière non exhaustive les modèles, les outils d'évaluations, les techniques de formation et de développement des compétences techniques et non techniques, du débriefing [11], voire des domaines tels que la relation entre le stress et la performance [12] et la rétention mémorielle des programmes pédagogiques [13,14]. Dans le domaine de la simulation, il ne devrait y avoir de pédagogie sans recherche comme il ne pourrait exister de recherche sans pédagogie. Celle-ci devrait être valorisée à la fois en termes de financement, mais aussi de publications scientifiques, encore trop difficilement acceptées. L'intégration ou l'association à des unités de recherche de l'Inserm ou du CNRS pourrait contribuer à l'essor de la recherche en simulation biomédicale. D'un point de vue de l'éducation, une réflexion doit être menée sur l'apprentissage en équipe pluriprofessionnelle. 
Des simulations incluant des internes de DES de médecine d'urgence associés à des étudiants en soins infirmiers devraient se développer afin de faciliter l'apprentissage des compétences non techniques et du travail en équipe. Cellesci sont source de la majorité des erreurs rencontrées lors de la gestion d'une situation de crise. Ce champ de compétence concerne le leadership, le travail d'équipe et la répartition des tâches, la qualité de la communication, la priorisation et la prise de décision, l'anticipation et la planification, la réévaluation et l'appel d'aide [15]. Enfin, au-delà de la formation initiale, se pose également la question de la formation médicale continue des urgentistes par simulation et de l'accessibilité des centres de simulation aux urgentistes exerçant en dehors des centres hospitalo-universitaires en secteur public ou privé.

La médecine d'urgence est devenue une spécialité, et la simulation doit accompagner son développement sur le plan universitaire. Il nous incombe de nous approprier pleinement cet outil et de jouer un rôle prépondérant dans le développement de la pédagogie et de la recherche en simulation. Il devrait y avoir un programme pédagogique uniforme, une accessibilité garantie à tous et une valorisation de la recherche en simulation.

\section{Références}

1. Okuda Y, Bond W, Bonfante G, et al (2008) National growth in simulation training within emergency medicine residency programs, 2003-2008. Acad Emerg Med 15:1113-6

2. Granry JC, Moll MC (2012) État de l'art (national et international) en matière de pratiques de simulation dans le domaine de la santé dans le cadre du développement professionnel continu (DPC) et de la prévention des risques associés aux soins. Rapport de Mission de la Haute Autorité de santé du 10 janvier 2012. www.has-sante.fr. (Dernier accès le 08 mars 2018)
3. Chakravarthy B, Ter Haar E, Bhat SS, et al (2011) Simulation in medical school education: review for emergency medicine. West J Emerg Med 12:461-6

4. Ghazali A, Ragot S, Breque C, et al (2016) Randomized controlled trial of multidisciplinary team stress and performance in immersive simulation for management of infant in choc: study protocol. Scand J Trauma Resusc Emerg Med 24:36

5. Allain M, Kuczer V, Longo C, et al (2018) Place de la simulation dans la formation initiale des urgentistes : enquête nationale observationnelle. Ann Fr Med Urgence 8:75-82

6. Riou B (2017) 2017 : l'an 1 du diplôme d'études spécialisées de médecine d'urgence. Ann Fr Med Urgence 7:1-4

7. Faudeux C, Tran A, Dupont A, et al (2017) Development of reliable and validated tools to evaluate technical resuscitation skills in a pediatric simulation setting: resuscitation and emergency simulation checklist for assessment in pediatrics. J Pediatr 188:252-7

8. Ghazali A, Leger A, Petitpas F, et al (2016) Development and validation of a performance assessment scale for chest tube insertion in traumatic pneumothorax. J Pulm Respir Medic 6:3

9. Oriot D, Bridier A, Ghazali A (2016) Team average performance assessment scale (TAPAS): how to evaluate team clinical performance. Health Care Curr Rev 4:164

10. Oriot D, Darrieux E, Boureau-Voultoury A, et al (2012) Validation of a performance assessment scale for simulated intraosseous access. Sim Healthcare 7:171-5

11. Rudolph JW, Simon R, Raemer DB, Eppich WJ (2008) Debriefing as formative assessment: closing performance gaps in medical education. Acad Emerg Med 15:1010-6

12. Ghazali DA, Darmian-Rafei I, Ragot S, et al (2018) Performance under stress conditions during multidisciplinary team immersive pediatric simulations. Pediatr Crit Care Med 19 (in press)

13. Boet S, Borges BC, Naik VN et al (2011) Complex procedural skills are retained for a minimum of $1 \mathrm{yr}$ after a single highfidelity simulation training session. Br J Anaesth 107:533-9

14. Yang CW, Yen ZS, McGowan JE, et al (2012) A systematic review of retention of adult advanced life support knowledge and skills in healthcare providers. Resuscitation 83:1055-60

15. Owen H, Mugford B, Follows V, Plummer JL (2006) Comparison of three simulation-based training methods for management of medical emergencies. Resuscitation 71:204-11 